\title{
The Disruption of a Gene Encoding a Putative Arylesterase Impairs Pyruvate Dehydrogenase Complex Activity and Nitrogen Fixation in Sinorhizobium meliloti
}

\author{
María José Soto, Juan Sanjuan, and José Olivares \\ Departamento de Microbiología del Suelo y Sistemas Simbióticos, Estación Experimental del Zaidín, CSIC, \\ Profesor Albareda, 1, 18008 Granada, Spain \\ Submitted 6 November 2000; 14 February 2001.
}

\begin{abstract}
Nitrogen-fixing Sinorhizobium meliloti cells depend upon dicarboxylic acids as carbon and energy sources. The metabolism of these intermediate compounds of the trichloroacetic acid cycle is dependent upon the availability of acetyl-coenzyme A (CoA). In bacteroids, the combined activities of malic enzymes and pyruvate dehydrogenase (PDH) have been proposed to be responsible for the anaplerotic synthesis of acetyl-CoA. We obtained a $S$. meliloti mutant strain, PD3, in which a Tn5 insertion led to a significant decrease in the overall PDH activity. The genetic characterization of this mutant revealed that the transposon is located at the $3^{\prime}$ end of a gene ( $a d a$ ) encoding a putative arylesterase. The mutant PD3 is deficient in nitrogen fixation, which strengthens the physiological importance of PDH activity in the symbiosis of $S$. meliloti with alfalfa plants.
\end{abstract}

Additional keywords: bacteroid metabolism, nodulation, succinate.

Bacteria of the family Rhizobiaceae (collectively referred to as "rhizobia") and legume plants have the ability to establish a symbiosis in which a specialized form of the bacteria (bacteroid) fix dinitrogen within a novel plant organ (root nodule). The establishment of a successful symbiosis requires the integration of the microbial metabolism with that of the host so that reduced carbon from the plant is exchanged for reduced nitrogen from the bacteria. $\mathrm{C}_{4}$-dicarboxylic acids such as malate and succinate generally have been considered the major carbon source exported from plant cells to the bacteroids to support the nitrogen-fixation process (Bolton et al. 1986). Mutant rhizobia that are impaired in $\mathrm{C}_{4}$-dicarboxylic acid transport form ineffective nodules. The metabolism of $\mathrm{C}_{4}$ dicarboxylates, acting as the sole carbon source via the trichloroacetic acid (TCA) cycle, is dependent upon the avail-

Corresponding author: J. Olivares; Telephone: +34 95812 1011; Fax: +34 95812 9600; E-mail: olivares@ eez.csic.es

Nucleotide and/or amino acid sequence data can be found at the EMBL database as accession no. AJ295059. ability of acetyl-coenzyme A (CoA). Bacteroids from alfalfa nodules lack phosphoenolpyruvate carboxykinase (PCK) activity (Finan et al. 1991). Thus, the pathway for the formation of acetyl-CoA via oxalacetate to phosphoenolpyruvate probably cannot operate in bacteroids. Instead, it has been proposed that the combined activities of malic enzymes and pyruvate dehydrogenase are responsible for the synthesis of acetylCoA in bacteroids (Driscoll and Finan 1993).

Malic enzymes convert malate to pyruvate and $\mathrm{CO}_{2}$ with the simultaneous reduction of $\mathrm{NAD}^{+}\left(\mathrm{P}^{+}\right)$. Two forms of malic enzymes that differ in cofactor requirement have been identified in Sinorhizobium meliloti cells, but only a functional $\mathrm{NAD}^{+}$malic enzyme is essential for symbiotic $\mathrm{N}_{2}$ fixation (Driscoll and Finan 1993; Driscoll and Finan 1996). The pyruvate dehydrogenase complex (PDHc) catalyzes the irreversible oxidative decarboxylation of pyruvate to acetyl-CoA, and it consists of multiple copies of three enzymes: pyruvate dehydrogenase (PDH) (E1p), dihydrolipoamide acetyltransferase (E2p), and dihydrolipoamide dehydrogenase (E3). Recently, three $p d h$ genes of the $S$. meliloti PDHc have been isolated and characterized: $p d h A \alpha$ and $p d h A \beta$, encoding the E1p component, and $p d h B$, encoding the E2p component (Cabanes et al. 2000). In contrast to the substrate-specific E1p and E2p, the E3 component, encoded by the lpd gene, is a common component of all 2-oxo acid dehydrogenase complexes. Accordingly, the lpd gene is in gram-negative bacteria as part of different gene clusters (De Kok et al. 1998). In the $S$. meliloti genome, $p d h A \alpha \beta$ and $p d h B$ are clustered as they are in other prokaryotic genomes, with the exceptions of $Z y$ momonas mobilis (Neveling et al. 1998) and Rickettsia prowazekii (Andersson et al. 1998). No lpd gene could be found immediately downstream of $p d h B$ (Cabanes et al. 2000). Instead, an open reading frame (ORF) starting $51 \mathrm{bp}$ downstream $p d h B$ and displaying sequence similarity to an arylesterase from Agrobacterium radiobacter was identified.

Despite the assumed importance of PDH activity in bacteroids, which contributes to the generation of the acetyl-CoA essential for the TCA cycle operation, no $p d h$ mutant and, therefore, its corresponding symbiotic phenotype has not yet been described. Here, we report the isolation of a mutant defective in PDH activity, which is impaired in nitrogen fixation. 
During the screening of $S$. meliloti Tn5-induced mutants that were altered in their ability to produce cellulolytic activity, a mutant, PD3, showed a symbiotic phenotype that encouraged us to perform a further characterization. Wild-type $S$. meliloti GR4 (Casadesús and Olivares 1979) and its Tn5derived mutant were grown at $30^{\circ} \mathrm{C}$ in TY complex medium (Beringer 1974) or in defined minimal medium (Robertsen et al. 1981). Carbon and nitrogen sources added to the latter medium are specified for each experiment.

Alfalfa (Medicago sativa L.) plants were grown in a nitrogen-free medium, as described by Olivares et al. (1980). To test the symbiotic phenotype of the rhizobial strains, 20 individual plants were inoculated with $10^{6}$ cells. After inoculation, the number of nodulated plants and the number of nodules per plant were recorded daily. For acetylene reduction assays, alfalfa plants were grown in Leonard jars (Leonard 1943) containing vermiculite. Ethylene formation was detected with a Hewlett Packard 5890 gas chromatograph (Palo Alto, CA, U.S.A.). Additionally, the shoot dry weights of 1-month-old nodulated plants were determined.

The mutant strain PD3 showed delayed nodulation kinetics (Fig. 1A) and, during the first days after inoculation, induced fewer nodules per plant than the parental strain GR4 (Fig. 1B). At the end of the experiment, however, plants inoculated with PD3 showed a significantly higher number of nodules compared with those inoculated with the wild type, a common feature among poor-fixing root nodule bacteria. The white nodules induced by PD3 contain bacteroids (data not shown). PD3, however, is impaired in its nitrogen-fixation ability be- cause its acetylene reduction activity is approximately half that of the wild-type strain (Fig. 1C) and the shoot dry weight of plants inoculated with PD3 was fivefold lower than that of plants inoculated with the wild type (Fig. 1D).

Genetic characterization was performed with standard DNA techniques described previously by Sambrook et al. (1989). Sequencing was performed with an ABI 373 automated sequencer (Columbia, MD, U.S.A.). DNA sequence edition, translation, and analysis were performed with the OMIGA 1.1.3 software package (Oxford Molecular, Oxford, U.K.) and the BLAST program from the network service at the National Center for Biotechnology Information (Bethesda, MD, U.S.A.)

DNA hybridization studies with a Tn5-derived probe revealed that the PD3 mutant carried a single Tn5 insertion located into a 4-kb EcoRI fragment. This fragment containing the Tn5 insertion was cloned into pBluescript KS (pBS) (Stratagene, La Jolla, CA, U.S.A.) to yield pPD10 (Fig. 2A). From this construction, the 4.4-kb EcoRI-BamHI fragment containing the kanamycin resistance gene of $\mathrm{Tn} 5$ and the remaining 6-kb BamHI-EcoRI fragment were isolated and subcloned into pBS to yield plasmids pD142 and pD162, respectively. We used a primer derived from the Tn5 inverted repeat sequence to sequence the transposon-flanking DNA. The results indicated that the insertion in the mutant PD3 was located at the $3^{\prime}$ end of an ORF encoding a protein with striking homology (71\% identity and $83 \%$ similarity in an overlap of 207 amino acids) with the arylesterase from A. radiobacter (accession no. AF044683) encoded by the ada gene. The ORF
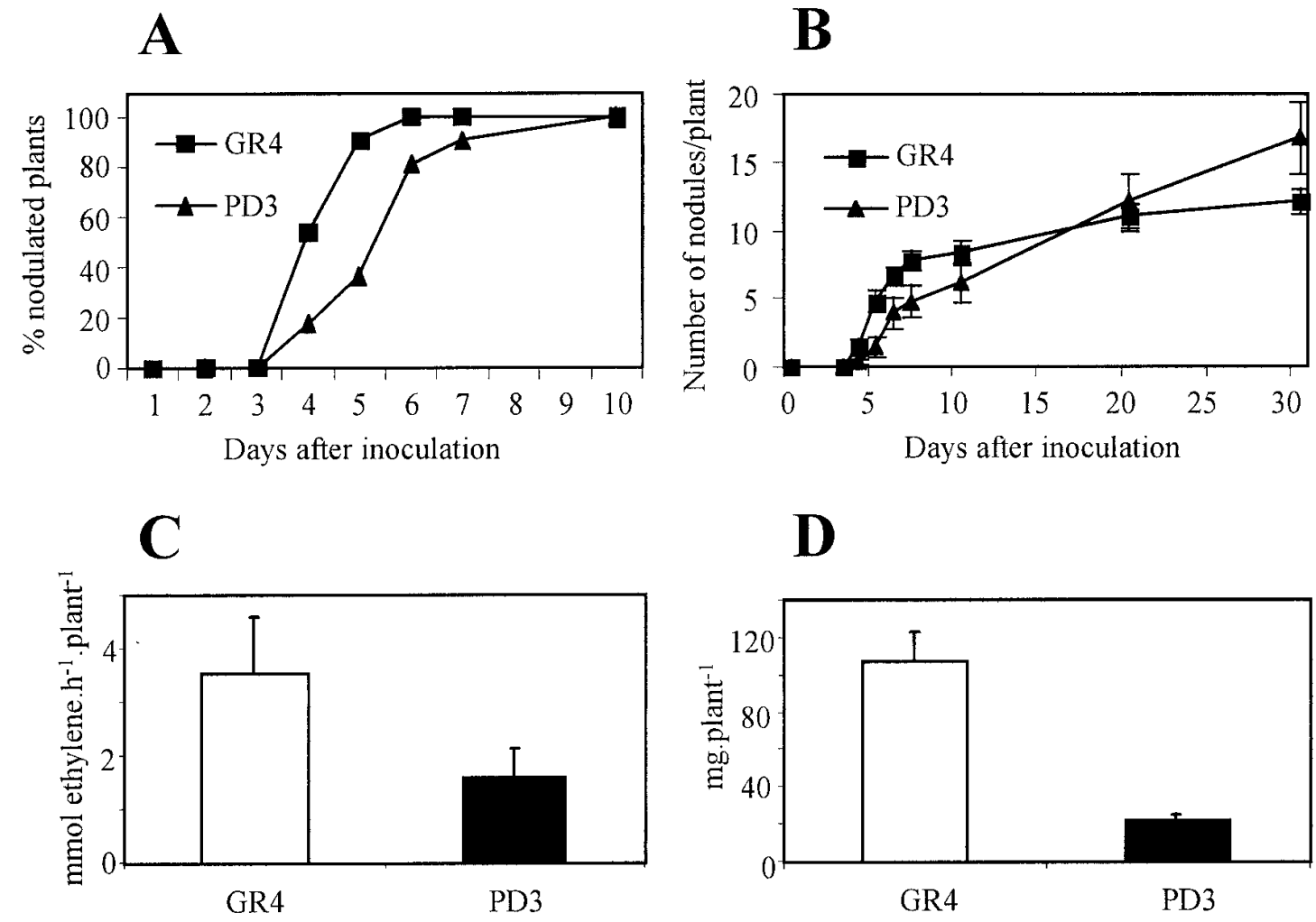

Fig. 1. Symbiotic phenotype of Sinorhizobium meliloti strain GR4 and the mutant derivative PD3. A, Nodulation kinetics expressed as percentage of nodulated plants. B, Number of nodules formed per plant. C, Acetylene reduction assay (ARA). D, Shoot dry weight of nodulated plants. Nodulation experiments were conducted three times with similar results. ARA and shoot dry weight data are means \pm standard error of values obtained with eight plants. 
putatively encoding this protein was inferred previously in $S$. meliloti 2011 by Cabanes and coworkers (2000), and is located $51 \mathrm{bp}$ downstream of the $p d h B$ gene. The amino acid sequence GDSLT, which has been defined as the active center motif of the arylesterase of Vibrio mimicus (Shaw et al. 1995) and other lipolytic enzymes (Upton and Buckley 1996), also is present in the amino terminal region of the $S$. meliloti protein. $A$. radiobacter arylesterase has been shown to be responsible for the conversion of 7-aminocephalosporanic acid (7-ACA) into deacetyl 7-ACA (Sakai et al. 1998). Interestingly, the ada gene encoding this arylesterase in Agrobacterium spp. is flanked at its $5^{\prime}$ and $3^{\prime}$ ends by genes encoding for putative dihydrolipoamide S-acetyltransferase and dihydrolipoamide dehydrogenase, respectively. The characterization of the remaining sequence present in the pD142 plasmid revealed that $600 \mathrm{bp}$ downstream of the $S$. meliloti ada gene there is an incomplete ORF displaying sequence similarity to the dihydrolipoamide dehydrogenase (E3) of the PDH multienzyme complexes from various sources. Therefore, we refer to this ORF as the lpd gene from $S$. meliloti. The sequence corresponding to the lpd gene obtained in this work and annotated at the EMBL database is partial, comprising 561 nucleotides from the $5^{\prime}$ end of the gene (Fig. 2A). To complete the sequence of the lpd gene, we used information from the $1-\mathrm{X}$ random pass of the $S$. meliloti 1021 genome performed by S. R. Long and coworkers at the Department of Biological Sciences, Howard Hughes Medical Institute (Chevy Chase, MD, U.S.A.); and the Stanford DNA Sequencing and Technology Center (Stanford, CA, U.S.A.). We used data corresponding to clones 423071A07, 423019C05, 423101E03, and 423081G02 to elongate the sequence by $1,282 \mathrm{bp}$, which was long enough to reach the putative stop codon of the lpd ORF. The analysis of the complete sequence revealed that the protein encoded by this gene shows up to $55 \%$ identity to the E3 component of the acetoin dehydrogenase complex of Klebsiella pneumoniae
(Peng et al. 1996) and 54 and 53\% identity to the E3 components of Z. mobilis (Neveling et al. 1998) and R. prowazekii (Andersson et al. 1998), respectively. The sequence contains the characteristic motifs of flavin-containing disulfide oxidoreductases (Williams 1992). This includes the flavin adenine dinucleotide and NAD binding sites as well as residues Cys 42 and Cys 47, which build the redox-active disulfide bridge involved in electron charge transfer with flavin adenine dinucleotide. In contrast to the genetic organization in $\mathrm{A}$. $\mathrm{ra}$ diobacter, we could identify an additional ORF (ORF1) in $S$. meliloti between the ada and the lpd genes, which putatively codes for a 194 amino acid protein of unknown function (Fig. 2A). In Z. mobilis, an additional ORF (ORF2) encoding a protein of 149 amino acids was identified between the structural genes $p d h B$ and $l p d$. Similarly, an ORF between the $p d h B$ and $l p d$ genes also was identified in the $p d h$ gene clusters of Alcaligenes eutrophus ( $p d h A-p d h B-\mathrm{ORF} 3-p d h \mathrm{~L})$ and Neisseria meningitidis (E1p-E2p-ORF3-E3). No similarity could be detected between the ORF2 of Z. mobilis or the ORF3 of A. eutrophus and N. meningitidis (Neveling et al. 1998) with the ORF1 of $S$. meliloti.

The small distances separating $a d a, \mathrm{ORF} 1$, and lpd (2 bp between $a d a$ and ORF1 and 17 bp between ORF1 and lpd) could be an indication that these three genes are cotranscribed. If so, then the Tn5 insertion present in the PD3 mutant could cause a polar effect on the expression of ORF1 and the E3 component of the PDH complex.

We analyzed the overall activity of the PDH complex and the E3 enzyme in crude cell extracts from wild-type GR4 and mutant strain PD3. Cell extracts were prepared from 100-ml samples of late-exponential phase cultures grown in mannitolMM supplemented with $20 \mathrm{mM}$ pyruvate. After centrifugation, the cells were resuspended in $50 \mathrm{mM}$ potassium buffer ( $\mathrm{pH}$ 7.0) containing $1 \mathrm{mM}$ EDTA and $50 \mu \mathrm{M}$ phenylmethylsulfonyl fluoride and disrupted in a French press. The suspen-
A
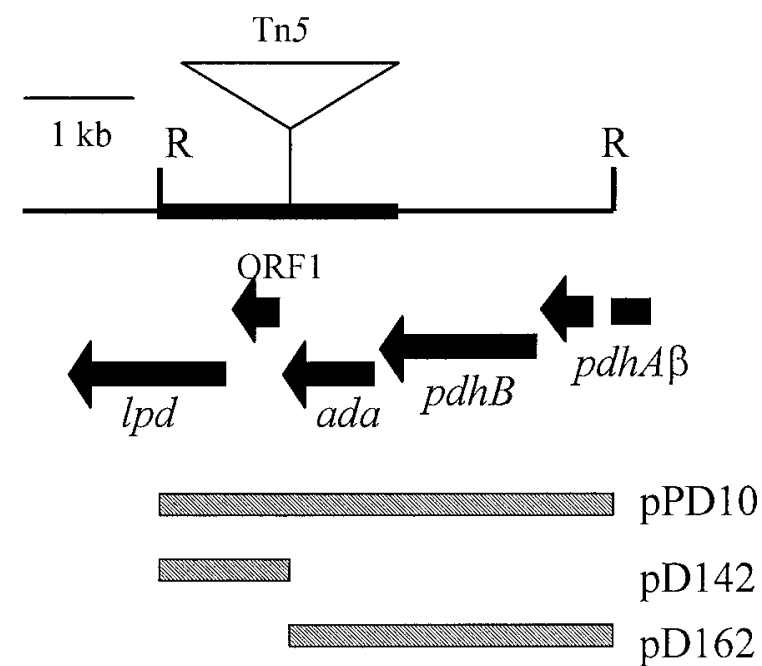

B

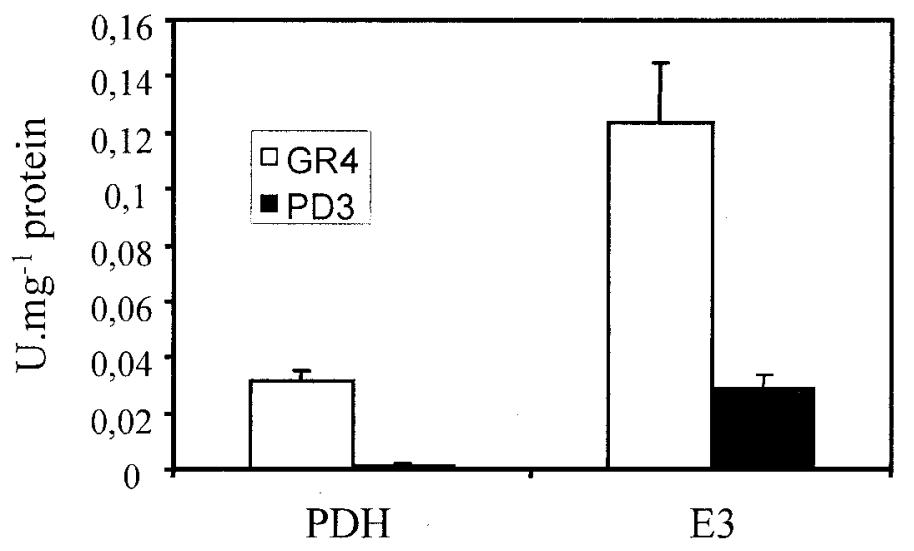

Fig. 2. A, Localization of the Tn5 insertion in the Sinorhizobium meliloti PD3 mutant. Thick line represents the sequence obtained in this work. Hatched boxes represent the genomic DNA present in the different plasmids. $p d h A \beta$, pyruvate dehydrogenase; $p d h B$, dihydrolipoamide acetyltransferase; $a d a$, arylesterase; lpd, dihydrolipoamide dehydrogenase; R, EcoRI. B, Pyruvate dehydrogenase (PDH) and dihydrolipoamide dehydrogenase (E3) enzyme activities in cell extracts from $S$. meliloti. 
sions were clarified by centrifugation at $4^{\circ} \mathrm{C}$. The resulting supernatants were used as cell extracts, and their protein contents were determined with the Bio-Rad (Hercules, CA, U.S.A.) protein assay reagent. The pyruvate dehydrogenase activity was measured spectrofotometrically at $340 \mathrm{~nm}$ and $25^{\circ} \mathrm{C}$ in $1-\mathrm{ml}$ cuvettes, as described previously (Jeyaseelan et al. 1980). The lipoamide dehydrogenase (E3; EC 1.6.4.3) component was determined as described previously (Reed and Willms 1966). Enzyme-specific activities are quoted in enzyme units per milligram of protein, where one unit is the amount of enzyme required to transform $1 \mu \mathrm{mol}$ substrate per $\min$.

The PDH activity in the extracts from PD3 was significantly lower (approximately 16-fold) than that observed in the extracts from wild-type strain GR4 (Fig. 2B). This result could be explained by the lower E3 activity detected in the extracts from the mutant strain. Thus, the Tn5 insertion in strain PD3 has an effect on the overall pyruvate dehydrogenase complex and E3 activity. It is noteworthy, however, that the reduction in the dihydrolipoamide dehydrogenase activity in PD3 was only fourfold, in contrast to the 16-fold reduction in the overall PDH activity. A possible explanation could be that $S$. meliloti harbors a second gene encoding a protein with dihydrolipoamide dehydrogenase activity. Comparison of the $S$. meliloti lpd-encoded protein with sequences corresponding to the $S$. meliloti genome-sequencing project revealed the existence of a similar 468 amino acid protein, displaying 39\% identity and 55\% similarity, which has been defined as the E3 component of the 2-oxoglutarate dehydrogenase (ODH) complex. The existence of a second protein with dihydrolipoamide dehydrogenase activity could explain the residual PDH and nitrogenase activities observed in the PD3 mutant as well as its ability to use succinate and pyruvate as the sole carbon sources, albeit at a reduced rate (data not shown).

The ODH complex catalyzes the oxidative decarboxylation of $\alpha$-ketoglutarate and is similar structurally to the PDH complex. Consequently, it also consists of three kinds of enzymes: an $\alpha$-ketoglutarate dehydrogenase component $\left(\mathrm{E}_{1}\right)$, a transsuccinylase $\left(\mathrm{E}_{2}\right)$, and a dihydrolipoyl dehydrogenase $\left(\mathrm{E}_{3}\right)$. In Escherichia coli, the PDH and ODH complexes share the lipoamide dehydrogenase subunit involved in the reoxidation of the E2-bound dihydrolipoate acid moiety (Mattevi et al. 1992). In $S$. meliloti, however, the sequence similarity data seem to indicate the existence of two different genes that code putatively for two different E3 components. Genetic characterization of this second gene and a biochemical characterization of its product are necessary to confirm whether the protein is functionally active as a lipoamide dehydrogenase.

The genetic characterization of our PDH mutant revealed that the insertion of the transposon is located at the $3^{\prime}$ end of the $a d a$ gene encoding a putative arylesterase. This ada gene is located between the $p d h B$ gene and a putative ORF (ORF1) that precedes the lpd gene. Cabanes et al. (2000) suggested that the $p d h A \alpha \beta-p d h B$ genes form a single transcriptional unit in $S$. meliloti. We could not identify any promoter-like structures upstream of $a d a$, ORF1, or $l p d$. Although we have no indication that $p d h B$ and $a d a-\mathrm{ORF} 1-l p d$ are transcribed as a single operon, the short distances between $p d h B$-ada-ORF1$l p d$, together with the significant reduction in dihydrolipoamide dehydrogenase activity (E3) shown by the PD3 mu- tant, tempt us to speculate that all these genes are cotranscribed and that the mutant phenotype probably is the result of the polar effect of the transposon insertion on the downstream lpd gene. We cannot exclude from our results, however, the possibility that the activity of the arylesterase, the putative ORF1-encoded protein, or both are necessary for the proper functioning of the PDH complex. Additional experiments are required to resolve this question.

Although PD3 is able to induce the formation of nodules that contain bacteroids, this mutant is less infective than the wild-type strain. More importantly, the nodules induced by the PDH mutant are deficient in nitrogen fixation. To our knowledge, this is the first time that the symbiotic phenotype of a mutant impaired in PDH activity has been reported. Furthermore, the results described here strengthen the physiological importance of this activity in bacteroid metabolism during symbiosis.

\section{ACKNOWLEDGMENTS}

We thank J. Nogales for help in acetylene reduction measurements. This work was supported by the Plan Andaluz de Investigación (PAI).

\section{LITERATURE CITED}

Andersson, S. G., Zomorodipour, A., Andersson, J. O., Sicheritz-Ponten, T., Alsmark, U. C., Podowski, R. M., Naslund, A. K., Eriksson, A. S. Winkelr, H. H., and Kurland, C. G. 1998. The genome sequence of Rickettsia prowazekii and the origin of mitochondria. Nature 396:133140.

Beringer, J. E. 1974. R factor transfer in Rhizobium leguminosarum. J. Gen. Microbiol. 84:188-198.

Bolton, E., Higginson, B., Harrington, A., and O'Gara, F. 1986. Dicarboxylic acid transport in Rhizobium meliloti: Isolation of mutants and cloning of dicarboxylic acid transport genes. Arch. Microbiol. 144:142-146.

Cabanes, D., Boistard, P., and Batut, J. 2000. Symbiotic induction of pyruvate dehydrogenase genes from Sinorhizobium meliloti. Mol. Plant-Microbe Interact. 13:483-493.

Casadesús, J., and Olivares, J. 1979. Rough and fine linkage mapping of the Rhizobium meliloti chromosome. Mol. Gen. Genet. 174:203-209.

De Kok, A., Hengeveld, A. F., Martin, A., and Westphal, A. H. 1998. The pyruvate dehydrogenase multi-enzyme complex from Gram-negative bacteria. Biochim. Biophys. Acta 1385:353-366.

Driscoll, B. T., and Finan, T. M. 1993. NAD(+)-dependent malic enzyme of Rhizobium meliloti is required for symbiotic nitrogen fixation. Mol. Microbiol. 7:865-873.

Driscoll, B. T., and Finan, T. M. 1996. NADP ${ }^{+}$-dependent malic enzyme of Rhizobium meliloti. J. Bacteriol. 178:2224-2231.

Finan, T. M., McWhinnie, E., Driscoll, B., and Watson, R. J. 1991. Complex symbiotic phenotypes result from gluconeogenic mutation in Rhizobium meliloti. Mol Plant-Microbe Interact. 4:386-392.

Jeyaseelan, K., Guest, J. R., and Visser, J. 1980. The pyruvate dehydrogenase complex of Pseudomonas aeruginosa PAO. Purification, properties and characterization of mutants. J. Gen. Microbiol. 120:393402.

Leonard, L. T. 1943. A simple assembly for use in testing of culture of rhizobia. J. Bacteriol. 45:523-527.

Mattevi, A., De Kok, A., and Perham, R. N. 1992. The pyruvate dehydrogenase complex. Curr. Opin. Struct. Biol. 2:277-287.

Neveling, U., Klasen, R., Bringer-Meyer, S., and Sahm, H. 1998. Purification of the pyruvate dehydrogenase multienzyme complex of $Z y$ momonas mobilis and identification and sequence analysis of the corresponding genes. J. Bacteriol. 180:1540-1548.

Olivares, J., Casadesús, J., and Bedmar, E. J. 1980. Method for testing degree of infectivity of Rhizobium meliloti strains. Appl. Environ. Microbiol. 56:389-393.

Peng, H. L., Deng, W. L., Yang, Y. H., and Chang, H. Y. 1996. Identification and characterization of the $a c o D$ gene encoding the dihydrolipoamide dehydrogenase of Klebsiella pneumoniae acetoin 
poamide dehydrogenase of Klebsiella pneumoniae acetoin dehydrogenase system. J. Biochem. 119:1118-1123.

Reed, L. J., and Willms C. R. 1966. Purification and resolution of the pyruvate dehydrogenase complex (Escherichia coli). Methods Enzymol. 9:247-265.

Robertsen, B. K., Aiman, P., Darvill, A. G., McNeil, M., and Alberstein, P. 1981. The structure of acidic extracellular polysaccharides secreted by Rhizobium leguminosarum and Rhizobium trifolii. Plant Physiol. 67:389-400.

Sakai, Y., Ayukawa, K., Yurimoto, H., Yamamoto, K., and Kato, N. 1998. A novel arylesterase active toward 7-aminocephalosporanic acid from Agrobacterium radiobacter IFO 12607: Purification and characterization. J. Ferment. Bioeng. 85:58-62.

Sambrook, J., Fritsch, E. F., and Maniatis, T. 1989. Molecular Cloning: A Laboratory Manual, 2nd ed. Cold Spring Harbor Laboratory, Cold Spring Harbor, NY, U.S.A.

Shaw, J. F., Chang, B. C., Chuang, K. H., Yen, Y. T., Wang, Y. J., and
Wang, F. G. 1995. Nucleotide sequence of a novel arylesterase gene from Vibrio mimicus and characterization of the enzyme expressed in Escherichia coli. Biochem. J. 298:675-680.

Upton, C., and Buckley, J. T. 1996. A new family of lipolytic enzymes? Trends Biochem. Sci. 20:177-178.

Williams, C. H., Jr. 1992. Lipoamide dehydrogenase, glutathione reductase, thioredoxin reductase, and mercuric ion reductase-A family of flavoenzyme transhydrogenases. Pages 121-211 in: Chemistry and Biochemistry of Flavoenzymes, Vol. 3. F. Müller, ed. CRC Press, Boca Raton, FL, U.S.A.

\section{AUTHOR-RECOMMENDED INTERNET RESOURCES}

Sinorhizobium meliloti 1X genome sequence, Howard Hughes Medical Institute (http://cmgm.stanford.edu/ mbarnett/1xgenome.htm). 